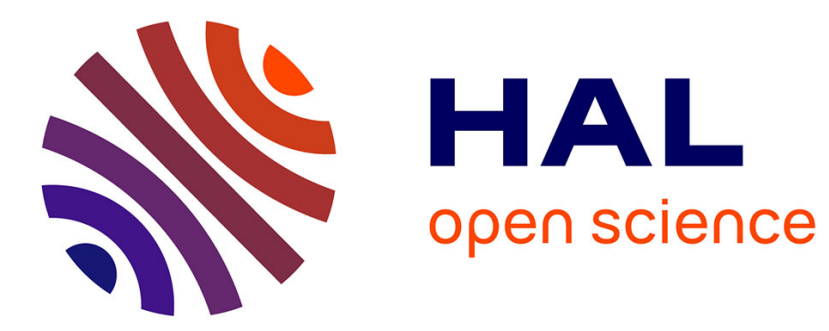

\title{
Reflecting Luneburg Lens: Analytical Solution and Applications
}

J. Ruiz-Garcia, E. Martini, C.D. Giovampaola, D. Gonzalez-Ovejero, S. Maci

\section{To cite this version:}

J. Ruiz-Garcia, E. Martini, C.D. Giovampaola, D. Gonzalez-Ovejero, S. Maci. Reflecting Luneburg Lens: Analytical Solution and Applications. 15th European Conference on Antennas and Propagation, EuCAP 2021, Mar 2021, Dusseldorf (virtual), Germany. pp.9411011, 10.23919/EuCAP51087.2021.9411011. hal-03249249

\section{HAL Id: hal-03249249 \\ https://hal.science/hal-03249249}

Submitted on 4 Jun 2021

HAL is a multi-disciplinary open access archive for the deposit and dissemination of scientific research documents, whether they are published or not. The documents may come from teaching and research institutions in France or abroad, or from public or private research centers.
L'archive ouverte pluridisciplinaire HAL, est destinée au dépôt et à la diffusion de documents scientifiques de niveau recherche, publiés ou non, émanant des établissements d'enseignement et de recherche français ou étrangers, des laboratoires publics ou privés. 


\title{
Reflecting Luneburg Lens: Analytical Solution and Applications
}

\author{
Jorge Ruiz-García*, Enrica Martini ${ }^{\dagger}$, Cristian Della Giovampaola ${ }^{\ddagger}$, \\ David González-Ovejero*, Stefano Maci ${ }^{\dagger}$ \\ *Univ Rennes, CNRS: Institut d'Électronique et de Télécommunications de Rennes (IETR), UMR 6164, \\ 35000 Rennes, France, \{jorge.ruiz, david.gonzalez-ovejero\}@univ-rennes1.fr \\ ${ }^{\dagger}$ University of Siena: Department of Information Engineering and Mathematics, \\ 53100 Siena, Italy, \{martini, macis\}@dii.unisi.it \\ ‡Wave Up s.r.l., 53100 Siena, Italy, cdg@wave-up.it
}

\begin{abstract}
This paper presents the closed-form solution for a new type of lens beamformer, which consists of two stacked parallel plate waveguides (PPWs) of circular shape. The rays launched by a source at the rim of the bottom PPW are collimated in the top PPW after being reflected at a cylindrical metallic wall that surrounds both layers. Since it reproduces the behavior of the Luneburg lens for reflected rays, this lens is hereinafter referred to as Reflecting Luneburg lens (RLL). The refractive index profile for the azimuthally symmetric circular domain is found by solving the non-linear integral equation of raycongruence through a truncated Abel transform method. The validity of the derived exact formula is verified by full-wave simulations, practical implementation and possible applications of RLLs in antenna systems are also briefly discussed.
\end{abstract}

Index Terms-Beamforming, flat optics, Luneburg lens, surface wave.

\section{INTRODUCTION}

Flat Luneburg lenses have been pervasively studied in the literature [1], [2], [3] as a compact solution for the generation of fan beams. The main advantage of Luneburg lenses is that one can steer the beam without suffering scan losses by changing the position of the source in the focal circumference. More recently, Luneburg lenses have been also used as beamformers for the excitation of modulated metasurfaces [4]. Such structure enables two-dimensional scanning of a pencil beam in the upper half-space. However, the Luneburg lens and the radiating aperture are at the same level in this architecture, so the total antenna footprint increases and the overall aperture efficiency is reduced.

To solve this problem, one can resort to pillbox quasi-optical beamformers [5], [6], which consist of two stacked parallel plate waveguides (PPWs). Both PPWs are connected through a $180^{\circ}$ bend with parabolic profile, so a source in the focus of the parabola generates a plane-wave in the top PPW. This plane wave can be gradually radiated using a slotted array [5], [6] or a modulated metasurface [7]. Despite the elegance of this approach, the scanning performance of the parabolic reflector degrades (deterioration in side-lobe levels arising from coma phase errors) when the source is displaced from the focal point to steer the beam.

The aforementioned shortcomings could be solved if one could reproduce the behavior of the Luneburg lens for rays

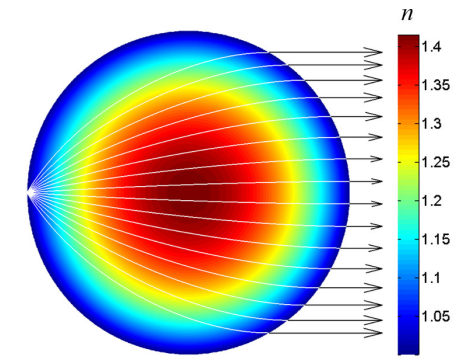

(a)

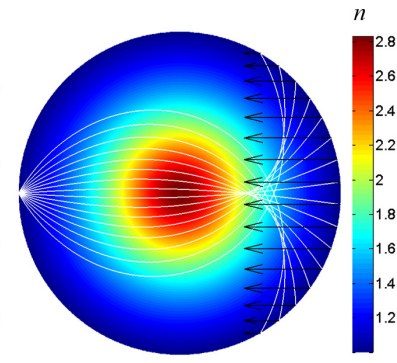

(b)
Fig. 1. Comparison between (a) Luneburg Lens and (b) Reflecting Luneburg Lens (RLL). In the RLL, the rays emerging from a point at the periphery undergo longer paths and, after reflecting at the boundary, they are coupled to an upper uniform guiding layer, where they form a plane wave.

reflected in a cylindrical wall of perfect electric conductor (PEC). The main contribution of this paper is the analytical derivation of the refractive index of such device, hereinafter referred to as Reflecting Luneburg Lens (RLL). Anticipating the result in Section III, Fig. 1(b) shows the refractive index profile and the ray-paths in a RLL. The rays launched by a source at the rim of the bottom PPW (white lines) are collimated in the top PPW after being reflected at a cylindrical PEC wall that surrounds both layers.

This paper is structured as follows. Section II describes the ray-optics problem. In Section III, the non-linear integral equation of ray-congruence is solved through a truncated Abel transform method. The analytical refractive index profile derived in the previous sections is validated in Section IV by full-wave simulations, and Section $\mathrm{V}$ presents one of the possible applications of RLLs in antenna systems. Finally, conclusions are drawn in Section VI.

\section{RAY OPTICS FORMULATION AND EXPRESSION FOR THE RAY-PATHS}

The following analysis stands for circular apertures of radius $R$, and a cylindrical reference system $(\rho, \phi, z)$ centered in the circular area. The problem at hand consists in determining the refractive index profile $n_{e q}(\rho)$ required in the bottom PPW to obtain a plane-wave in the top one. After simplifying (11) in 


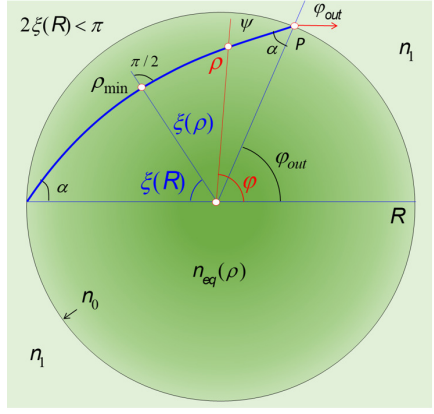

(a)

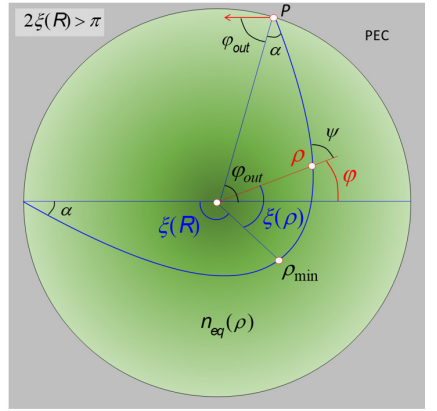

(b)
Fig. 2. Ray-path in a PPW filled with a radially graded index medium for two cases: (a) $2 \xi(R)<\pi$, (b) $2 \xi(R)>\pi$. Case (a) represents the ray-path in a conventional Luneburg lens and case (b) represents the ray-path in a RLL, when a PEC wall is around the lens rim and reflected rays are canalized in an upper PPW with refractive index $n_{1}$.

[8] for the case of an azimuthally symmetric medium, one can write:

$$
\frac{1}{\rho} \frac{d}{d \ell}\left[n_{e q} \rho^{2} \frac{d \varphi}{d \ell}\right]=0
$$

where $d \ell$ is the elementary length along a curvilinear ray-path. From (1), it is clear that $n_{e q} \rho^{2} d \varphi / d \ell$ will be constant along the ray-path (except at $\rho=0$ ). To further simplify the calculation of the ray trajectory, the angle $\psi$ is defined between the ray direction and the radial coordinate (see Fig. 2) such that

$$
\begin{aligned}
d \rho / d \ell & =\cos \psi \\
\rho d \varphi / d \ell & =\sin \psi
\end{aligned}
$$

Substituting (2b) in the expression between brackets in (1), one obtains the generalization of the Snell's law for radially graded index (GRIN) medium

$$
n_{e q} \rho \sin \psi=L,
$$

where $L$ is constant along the ray-path and it can be interpreted as a ray angular momentum.

Furthermore, one can divide (2b) by (2a) and use (3) to write the ray-path trajectory just as a function of $\rho, \phi$ as

$$
\rho \frac{d \varphi}{d \rho}=\mp \frac{L}{\sqrt{\left(\rho n_{e q}(\rho)\right)^{2}-L^{2}}}
$$

where the upper/lower sign applies for $\psi<\frac{\pi}{2}, \psi>\frac{\pi}{2}$.

Let us now consider a source placed at $\left(\rho_{0}, \varphi_{0}\right)=(R, \pi)$, as illustrated in Fig. 2, and denote by $\alpha$ the angle between the ray departing from the source and the $x$-axis. Since $L$ is constant on the ray-path, it can be calculated as $L=R n_{0} \sin \alpha$, where $n_{0}$ is the refractive index at the rim of the lens. From (3), one can also define $\rho_{\min }$ as the minimum radial distance for the trajectory of a given ray. This quantity satisfies $n_{e q}\left(\rho_{\min }\right) \rho_{\min }=L$ and it corresponds to the point where $\psi=\pi / 2$. Since a ray cannot propagate when its momentum is larger than $L$, the ray undergoes a turning point at $\rho_{\text {min }}$, $\psi$ is larger than $\pi / 2$ before the turning point and it becomes smaller after. To determine the ray-path after the turning point, it is convenient to define the positive angle $\xi(\rho)$ [shown in Fig. 2(a) and (b)] as

$$
\xi(\rho)=\int_{\rho_{\text {min }}}^{\rho} \frac{|L|}{\rho^{\prime} \sqrt{\left(\rho^{\prime} n_{e q}\left(\rho^{\prime}\right)\right)^{2}-L^{2}}} d \rho^{\prime},
$$

Bearing in mind the symmetry of the trajectory around the turning point, one can write the azimuthal angle covered by the ray until the observation point as $\xi(R)+\operatorname{sgn}\left(\frac{\pi}{2}-\psi\right) \xi(\rho)$. In turn, the total angle covered by the ray is $2 \xi(R)$.

Two different cases can be individuated for the ray-path depending on if $2 \xi(R)$ is larger or smaller than $\pi$ :

$$
\begin{aligned}
& \varphi(\rho)=\pi-\left(\xi(R)+\operatorname{sgn}\left(\frac{\pi}{2}-\psi\right) \xi(\rho)\right) ; \quad 2 \xi(R)<\pi . \\
& \varphi(\rho)=-\pi+\left(\xi(R)+\operatorname{sgn}\left(\frac{\pi}{2}-\psi\right) \xi(\rho)\right) ; \quad 2 \xi(R)>\pi .
\end{aligned}
$$

In $(6 a), \varphi \in[0, \pi)$, whereas in $(6 \mathrm{~b}) \varphi \in(-\pi, \pi)$. Equations (6a) $-(6 b)$ give the explicit functional equation in polar coordinates $(\rho, \varphi)$ of the ray trajectory of momentum $L$ for a given $n_{e q}(\rho)$ profile.

One can also observe that, due to the symmetry of the problem, the ray ends its trajectory inside the lens with the same angle $\alpha$ with which it is launched from the source. This property stems from the conservation of the angular momentum. Therefore, at the exit point $P$ the angle $\varphi$ assumes the values

$$
\varphi_{\text {out }}= \pm(\pi-2 \xi(R)) \text { for } 2 \xi(R) \lessgtr \pi .
$$

The ray-paths given by (6a) and (6b) represent two quite different situations, illustrated in Fig. 2(a) and Fig. 2(b), respectively. When $2 \xi(R)<\pi$ (6a) the behavior is that of the Luneburg lens, and it is possible to collimate outgoing rays parallel to the $x$-axis. Conversely, when $2 \xi(R)>\pi(6 \mathrm{~b})$, one can obtain ray congruence in reflection, assuming that a cylindrical PEC wall surrounds the rim of the lens. The latter behavior is the one sought for the RLL. Applying Snell's law at the circumference $\left(n_{0} \sin \alpha=n_{1} \sin \varphi_{\text {out }}\right)$ and using (7) and (5), one can write

$\pi \pm \arcsin \left(\frac{n_{0}}{n_{1}} \sin |\alpha|\right)=2 \int_{\rho_{\min }}^{R} \frac{\sin |\alpha|}{\rho^{\prime} \sqrt{\left(\rho^{\prime} n_{e q} / R n_{0}\right)^{2}-\sin ^{2} \alpha}} d \rho^{\prime}$,

where the lower sign corresponds to the condition for the Luneburg lens, whereas the uper one stands for the RLL.

\section{EXACT SOLUTION FOR THE REFLECTING LUNEBURG LENS}

Equation (8) is solved in closed-form when $n_{0}=n_{1}$ using the truncated Abel transform method. To that end, we normalize the radius $(R=1)$ and define the new variables $N(\rho)=n_{e q}(\rho) \rho / n_{0}$ and $l=\sin \alpha$ with $l \in(0,1)$, so $N(1)=1$ and $N\left(\rho_{\min }\right)=l$. Upon substitution in (8) one has

$$
\pi+\arcsin (l)=2 \int_{\rho_{\min }}^{1} \frac{l}{\rho \sqrt{(N(\rho))^{2}-l^{2}}} d \rho .
$$

Next, we apply the change of variables $x^{\prime}=\ln \rho$ and use $\eta\left(x^{\prime}\right)=N\left(e^{x^{\prime}}\right)$ in (9), thus obtaining

$$
\pi+\arcsin (l)=2 \int_{\ln \rho_{\min }}^{0} \frac{l}{\sqrt{\eta^{2}\left(x^{\prime}\right)-l^{2}}} d x^{\prime} .
$$




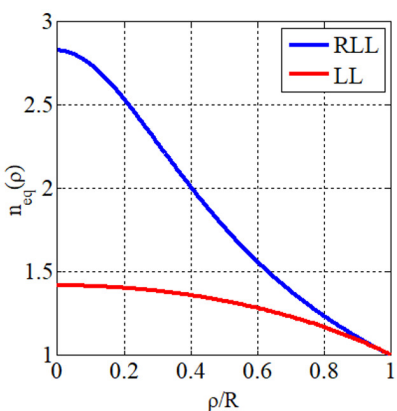

(a)

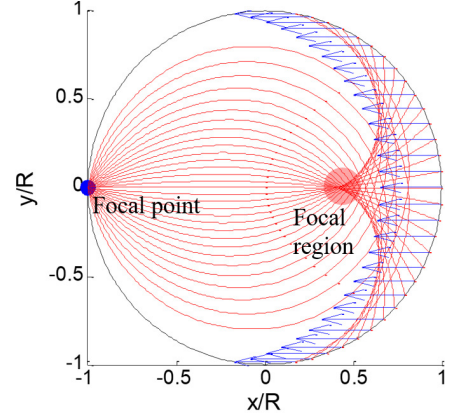

(b)
Fig. 3. (a) Radial profile of the refractive index of the RLL (blue line) and of the equivalent Luneburg Lens (red line) for $n_{0}=n_{1}=1$. (b) Ray-paths of the RLL (red lines) and direction of reflected rays in upper PPW (blue arrows).

A second change of variables, $\eta^{\prime}=\eta\left(x^{\prime}\right)$, is applied in (10). Since $x^{\prime}=x\left(\eta^{\prime}\right),(10)$ may be written as

$$
\pi+\arcsin (l)=2 \int_{l}^{1} \frac{d x^{\prime}}{d \eta^{\prime}} \frac{l}{\sqrt{\eta^{\prime 2}-l^{2}}} d \eta^{\prime},
$$

where we have used $N(1)=1$ in the upper limit of the integral. The left-hand side of (11) is the truncated Abel transform of the inverse function $\eta^{\prime}=\eta\left(x^{\prime}\right)$. After applying the inverse Abel transform [8] to both members, one obtains

$$
\int_{\eta}^{1} \frac{\pi+\arcsin \left(l^{\prime}\right)}{\sqrt{l^{\prime 2}-\eta^{2}}} d l^{\prime}=-\pi \ln \rho .
$$

The sum of integrals in the left-hand side of (12) can be written in closed form as in [8]. Upon substitution in (12), one has

$$
\ln \left(\frac{1+\sqrt{1-\eta^{2}}}{\eta}\right)+\frac{1}{2} \ln \left(1+\sqrt{1-\eta^{2}}\right)=-\ln \rho .
$$

Considering that $\eta(\ln \rho)=N(\rho)=n_{e q}(\rho) \rho / n_{0}$, the inversion of (13), after re-normalizing by $R$, leads to

$$
n_{e q}=n_{0}\left(\frac{-1+\sqrt{1+8(\rho / R)^{2}}}{2(\rho / R)^{2}}\right)^{3 / 2},
$$

which is the exact form of the refractive index profile of the RLL. Although the condition in (8) for Luneburg lenses and RLLs simply differs in a sign, the RLL refractive index profile in (14) is quite different from that of the Luneburg lens $n_{e q}=n_{0} \sqrt{2-(\rho / R)^{2}}$. Fig. 3(a) compares the radial profile of the refractive index for the RLL (blue line) with that of the Luneburg lens (red line). The ray-paths for the RLL are shown in Fig. 3(b), where the red and blue lines represent the rays in the bottom and top PPWs, respectively. It is also important to note that an inner focal region arises around the point $(\rho, \phi)=(0.46 R, 0)$; this fact will be exploited in Section V.

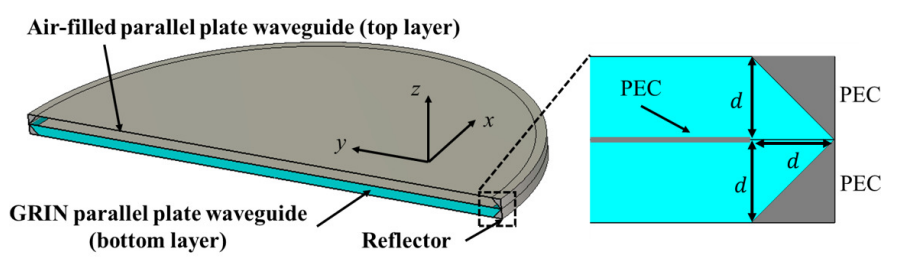

(a)

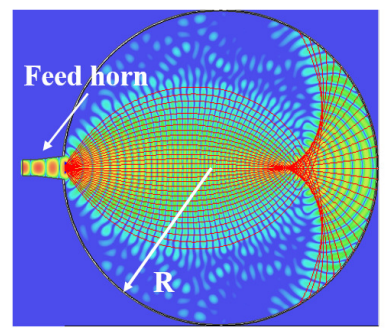

(b)

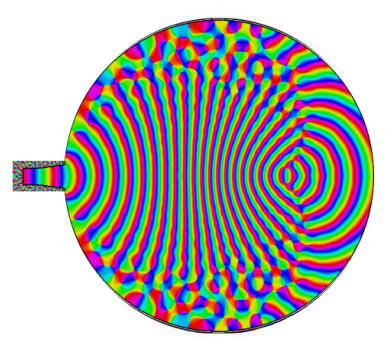

(d)

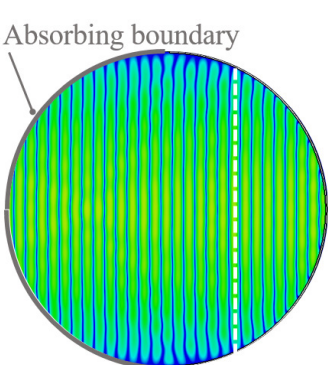

(c)

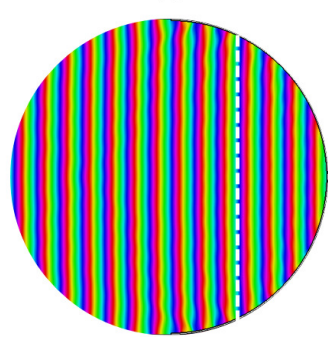

(e)
Fig. 4. (a) Perspective view of simulated RLL cutted in the $y z$ plane, and inset with detail of the corner reflector. Real part of the vertical component of the electric field in logarithmic scale for (b) the bottom and (c) the top PPWs. Phase distribution in degrees for (d) the bottom and (e) the top PPWs.

\section{NumericAl RESUlTS}

The performance of the RLL is verified in this section by full-wave simulations in CST Microwave Studio [9]. The simulated structure, shown in Fig. 4(a), consists of two stacked circular PPWs with radius $R=7 \lambda_{0}$, with $\lambda_{0}$ being the freespace wavelength. The bottom PPW is filled with a variably dielectric medium with the radial refractive index profile given by (14) and plotted in Fig. 3(a). Both parallel plates have a thickness $d=0.15 \lambda_{0}$ and are connected by a $180^{\circ}$ bend implemented by a corner reflector with the geometry presented in the right inset of Fig. 4(a). Fig. 4(b) and (c) show the amplitude of the vertical component of the electric field in the bottom and top PPWs, respectively. On the other hand, Fig. 4(d) represents the corresponding phase in the bottom PPW, whereas Fig. 4(e) depicts the phase in the top PPW. One can observe that a plane-wave is neatly formed in the top PPW, thus achieving the desired behavior. Given the azimuthal symmetry of the GRIN profile, it is clear that one avoids phase errors when moving the source around the focal circumference, as opposed to pillbox systems based on parabolic reflectors.

\section{APPLICATION IN ANTENNA SYSTEMS}

In addition to their potential for eliminating the scan loss in pillbox systems, RLLs can be also used as beamformers 


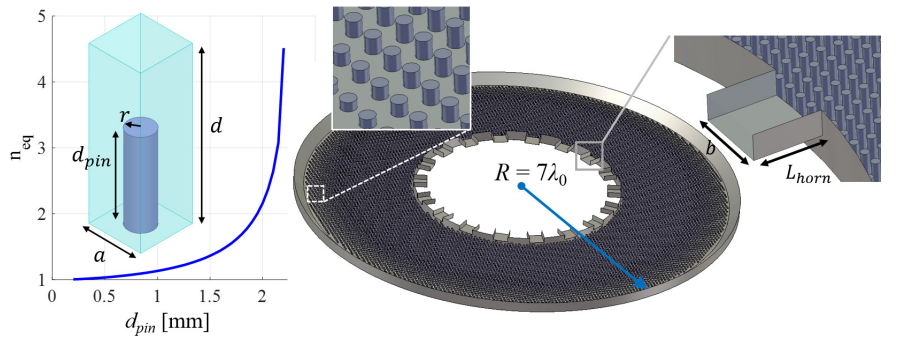

Fig. 5. Left: Mapping between the height of a metallic pin and equivalent refractive index where $r=0.3 \mathrm{~mm}, a=1.25 \mathrm{~mm}$, and $d=3.75 \mathrm{~mm}$ for an operating frequency of $30 \mathrm{GHz}$. Right: Bottom PPW for the multibeam RLL beamformer implemented by a bed of nails, the radius of the structure is $R=7 \lambda_{0}$, where $\lambda_{0}$ is the free-space wavelength at $30 \mathrm{GHz}$.

enabling a complete azimuthal coverage. To that end, instead of placing the primary feed at the focal circumference, one can put it in the focal region highlighted in Fig. 3(b). Fig. 5 depicts the bottom PPW of the proposed structure with multiple excitation ports. It is to be observed that, in this case, the variable dielectric has been implemented by metallic pins, as suggested in [10], [11]. The correspondence between the pins' height and the equivalent refractive index is shown in the left inset in Fig. 5 for a simulation frequency of $30 \mathrm{GHz}$. The top parallel plate has been also replaced by an open structure consisting of a metasurface sinusoidally modulated in the $x$ direction, implemented likewise by a bed of nails (see inset on left side of Fig. 6(a)). Fig. 6 also shows the 3D directivity patterns and color-maps in the reciprocal $(u-v)$ plane for sources placed at $\phi=0^{\circ}, \phi=20^{\circ}$ and $\phi=40^{\circ}$. The azimuth and elevation angles of the pencil beam change as one switches ports.

The obtained operation is similar to that of Variable Inclination Continuous Transverse Stub (VICTS) array, where the beam is steered by changing the relative orientation of a planar wavefront and a slotted plate [12].

\section{CONCLUSION}

This paper presents the analytical derivation of the refractive index profile for the Reflecting Luneburg lens (RLL). It consists of two stacked parallel plate waveguides (PPWs) with circular shape. The refractive index profile in the bottom PPW is such that the rays are collimated in the top one, after being reflected at the cylindrical PEC wall. To the best of our knowledge, despite their simple expression, RLLs have never been studied before. The validity of the derived close-form formula has been verified by full-wave simulations and possible applications in antenna systems have been also discussed.

\section{ACKNOWLEDGMENT}

The work of E. Martini and S. Maci was supported in part by the European Space Agency, under contract no. $4000127381 / 19 / \mathrm{NL} / \mathrm{AF}$, and in part by the Italian Ministry of University and Research, under the PRIN project "Metasurface Antennas for Satellite Applications". The work of J. RuizGarcía and D. González-Ovejero was funded in part by the
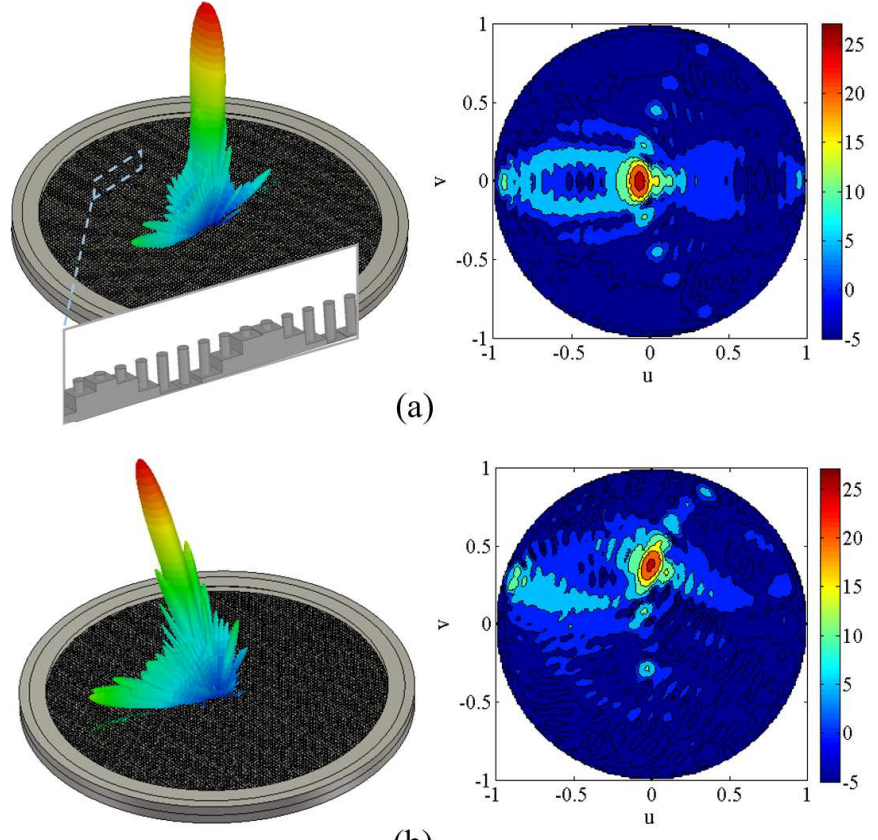

(b)
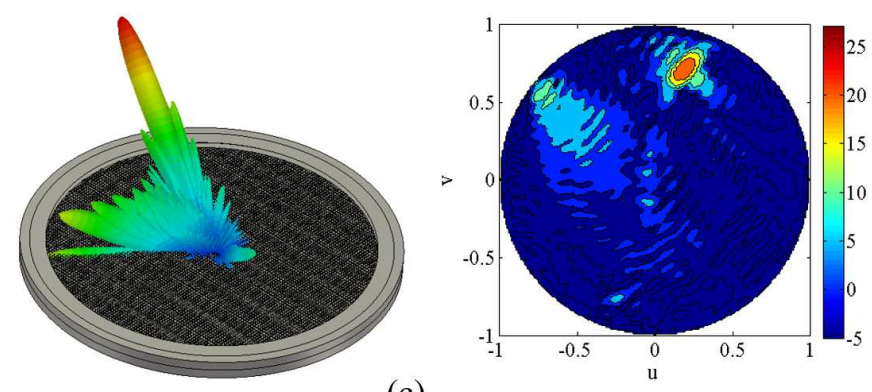

(c)

Fig. 6. 3D view of the radiated beam (left column) and u-v color maps (right column) showing the directivity in $\mathrm{dBi}$ when the angle of the excited horn in Fig. 5 with the $x$-axis is (a) $0^{\circ}$, (b) $20^{\circ}$ and (c) $40^{\circ}$.

European Union through the European Regional Development Fund (ERDF), and by the French Region of Brittany, Ministry of Higher Education and Research, Rennes Métropole and Conseil Départemental 35, through the CPER Project STIC $\&$ Ondes. They also received the support of the Agence de l'Innovation de Défense (AID), the École des Docteurs de l'UBL (Université Bretagne Loire) and the Conseil Régional de Bretagne.

\section{REFERENCES}

[1] Young-Jin Park, A. Herschlein, and W. Wiesbeck, "A photonic bandgap (PBG) structure for guiding and suppressing surface waves in millimeterwave antennas," IEEE Trans. Microw. Theory Techn., vol. 49, no. 10, pp. 1854-1859, Oct. 2001

[2] C. Pfeiffer and A. Grbic, "A printed, broadband Luneburg lens antenna," IEEE Trans. Antennas Propag., vol. 58, no. 9, pp. 3055-3059, Sep. 2010.

[3] M. Bosiljevac, M. Casaletti, F. Caminita, Z. Sipus, and S. Maci, "Non-uniform metasurface Luneburg lens antenna design," IEEE Trans. Antennas Propag., vol. 60, no. 9, pp. 4065-4073, Sep. 2012.

[4] Y. B. Li, R. Y. Wu, W. Wu, C. B. Shi, Q. Cheng and T. J. Cui, "Dual-Physics Manipulation of Electromagnetic Waves by System-Level Design of Metasurfaces to Reach Extreme Control of Radiation Beams,' Adv. Mater. Technol., vol. 2, no. 1, p. 1600196, 2017. 
[5] M. Ettorre, R. Sauleau, L. Le Coq, and F. Bodereau, "Single-folded leaky-wave antennas for automotive radars at $77 \mathrm{GHz}$,' IEEE Antennas Wireless Propag. Lett., vol. 9, pp. 859-862, Sep. 2010.

[6] M. Ettorre, R. Sauleau, and L. Le Coq, "Multi-beam multi-layer leakywave SIW pillbox antenna for millimeter-wave applications," IEEE Trans. Antennas Propag., vol. 59, no. 4, pp. 1093-1100, Apr. 2011.

[7] J. Ruiz-García, M. Faenzi, A. Mahmoud, M. Ettorre, P. Potier, P. Pouliguen, R. Sauleau, and D. González-Ovejero, "Quasi-optical excitation of a circularly-polarized metasurface antenna at K-band," in 14th Eur. Conf. Antennas Propag. (EuCAP), 2020, pp. 1-4.

[8] S. Cornbleet, "Geometrical optics reviewed: A new light on an old subject," Proc. IEEE, vol. 71, no. 4, pp. 471-502, Apr. 1983.

[9] CST of America, "CST Microwave Studio," Anaheim, CA, 2019.
[10] D. González-Ovejero, C. Jung-Kubiak, M. Alonso-delPino, T. Reck, and G. Chattopadhyay, "Design, fabrication and testing of a modulated metasurface antenna at $300 \mathrm{GHz}$," in Proc. 11th Eur. Conf. Antennas Propag. (EUCAP), Paris, France, Mar. 19-24 2017, pp. 3416-3418.

[11] D. González-Ovejero, N. Chahat, R. Sauleau, G. Chattopadhyay, S. Maci, and M. Ettorre, "Additive manufactured metal-only modulated metasurface antennas," IEEE Trans. Antennas Propag., vol. 66, no. 11, pp. 6106-6114, Nov. 2018

[12] R. S. Hao, Y. J. Cheng, and Y. F. Wu, "Shared-aperture variable inclination continuous transverse stub antenna working at K- and Ka-bands for mobile satellite communication," IEEE Trans. Antennas Propag., vol. 68, no. 9 , pp. 6656-6666, 2020 